\title{
Magnetically Induced Optical Transparency on a Forbidden Transition in Strontium for Cavity-Enhanced Spectroscopy
}

\author{
Matthew N. Winchester, Matthew A. Norcia, Julia R. K. Cline, and James K. Thompson \\ JILA, NIST, and Department of Physics, University of Colorado, 440 UCB, Boulder, Colorado 80309, USA
}

(Received 9 January 2017; published 26 June 2017)

\begin{abstract}
In this Letter we realize a narrow spectroscopic feature using a technique that we refer to as magnetically induced optical transparency. A cold ensemble of ${ }^{88} \mathrm{Sr}$ atoms interacts with a single mode of a high-finesse optical cavity via the $7.5 \mathrm{kHz}$ linewidth, spin forbidden ${ }^{1} S_{0}$ to ${ }^{3} P_{1}$ transition. By applying a magnetic field that shifts two excited state Zeeman levels, we open a transmission window through the cavity where the collective vacuum Rabi splitting due to a single level would create destructive interference for probe transmission. The spectroscopic feature approaches the atomic transition linewidth, which is much narrower than the cavity linewidth, and is highly immune to the reference cavity length fluctuations that limit current state-of-the-art laser frequency stability.
\end{abstract}

DOI: 10.1103/PhysRevLett.118.263601

There has been a dedicated effort in recent years to improve the frequency stability of lasers [1] used to probe optical atomic clocks [2-4]. Improvements in these precision measurement technologies are essential for advancing a broad range of scientific pursuits such as searching for variations in fundamental constants [5], gravitational wave detection [6,7], and physics beyond the standard model [8,9]. Associated improvements in atomic clocks would also advance recent work on relativistic geodesy [10].

The frequency stability of current state-of-the-art lasers is limited by thermal fluctuations in the reference cavity mirror coatings, substrates, and spacer [11]. This problem can be alleviated by creating systems that rely on an ensemble of atoms, rather than a reference cavity, to achieve stable optical coherence. Recent approaches include cavity-assisted nonlinear spectroscopy [12-14] and superradiant lasers [15-18]. Both approaches use narrow forbidden transitions with linewidths ranging from $7.5 \mathrm{kHz}$ to $1 \mathrm{mHz}$. These novel systems are absolute frequency references and are intrinsically less sensitive to both fundamental thermal and technical vibrations that create noise on the optical cavity's resonance frequency.

Here, we demonstrate a new linear spectroscopy approach in which a static magnetic field can induce optical transparency in the transmission spectrum of an optical cavity. The center frequency of the transparency window is shown to be insensitive to changes in the cavity-resonance frequency and to first-order Zeeman shifts. The observed linewidth of the feature approaches the natural linewidth of the $7.5 \mathrm{kHz}$ optical transition and can be insensitive to inhomogeneous broadening of the atomic transition frequency. The linewidth of the feature is an important attribute for laser stabilization, as a laser stabilized to a narrow spectroscopic feature is less sensitive to technical offsets than a laser stabilized to a broader feature. In the future, it might be possible to extend this technique to even narrower optical transitions for enhanced spectroscopic sensitivity in atoms such as calcium and magnesium.

In analogy to electromagnetically induced transparency (EIT) [19-21], we refer to this effect as magnetically induced transparency (MIT) [22]. In EIT, a control laser is used to create a variable-width transparency window for slowing light [24], for stopping light [25], for quantum memories [26], and even for creating effective photonphoton interactions [27-29]. It might be possible to utilize controlled magnetic fields and long-lived optical states to realize similar goals.

In our experiment we create a strongly coupled atomcavity system by loading up to $N=1.3 \times 10^{6}{ }^{88} \mathrm{Sr}$ atoms into a one-dimensional optical lattice supported by a highfinesse optical cavity. The peak trap depth is $100(10) \mu \mathrm{K}$ and the atoms are laser cooled to $10(1) \mu \mathrm{K}$ [see Fig. 1(a) and Refs. [16,17,30]]. We tune a TEM00 resonance of the cavity at frequency $\omega_{c}$ to be near resonance with the dipoleforbidden singlet to triplet optical transition ${ }^{1} S_{0}$ to ${ }^{3} P_{1}$ at frequency $\omega_{0}$ or wavelength $689 \mathrm{~nm}$ [see Fig. 1(b)]. The excited state ${ }^{3} P_{1}$ spontaneously decays back to the ground state at a rate $\gamma=2 \pi \times 7.5 \mathrm{kHz}$, and the cavity power decays at rate $\kappa=2 \pi \times 150.3(4) \mathrm{kHz}$. In ${ }^{88} \mathrm{Sr}$, the absence of nuclear spin means that the ${ }^{1} S_{0}$ ground state is unique, while the ${ }^{3} P_{1}$ excited state has three Zeeman sublevels [see Fig. 1(b)].

In the limit of zero applied magnetic field, our system responds to an applied probe as though each atom were a simple two-level system. The ensemble can collectively absorb light from and then collectively reemit light into the cavity mode at the so-called collective vacuum Rabi frequency $\Omega=\sqrt{N} 2 g$. Here, $2 g / 2 \pi=15 \mathrm{kHz}$ is the rms value of the single-atom vacuum Rabi frequency, which accounts for averaging over the standing-wave cavity 


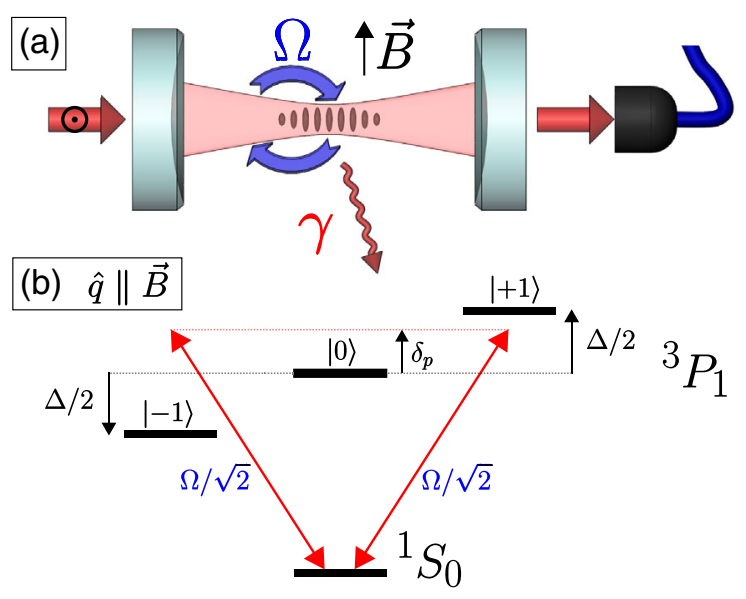

FIG. 1. (a) Simplified experimental diagram. The system is probed with horizontally polarized probe light. The light can be coherently absorbed by the atoms (the brown ovals) and reemitted into the cavity at collective vacuum Rabi frequency $\Omega$. The transmitted power is detected on a single photon counting module (black). A magnetic field is applied along the vertical direction. (b) The atomic energy level diagram of the ground ${ }^{1} S_{0}$ and excited states ${ }^{3} P_{1}\left|m_{j}\right\rangle$ with a quantization axis $\hat{q}$ parallel to the applied magnetic field $\vec{B}$. The applied magnetic field creates a Zeeman splitting $\Delta$ between the excited states $| \pm 1\rangle$. Both of these transitions interact equally with the horizontally polarized light inside the cavity with the collectively enhanced Rabi frequency $\Omega / \sqrt{2}$. The transmitted probe light is measured as the probe's detuning $\delta_{p}$ is swept. The $|0\rangle$ state is shown, but it does not interact with the horizontally polarized cavity field.

mode. This exchange creates two new transmission modes that are shifted away from the empty cavity's transmission peak by $\pm \Omega / 2$, as shown by the central red trace in Fig. 2(a).

Because two orthogonal components of the probe light can couple to two distinct Zeeman sublevels, the coupled atomcavity system has three normal modes of excitation, not two [see Figs. 1(b) and 1(c)]. In addition to the two modes at $\pm \Omega / 2$ that lead to the transmission peaks, which we will refer to as the "bright" modes, there is a third "dark" mode whose frequency is equal to that of the atomic transition. The dark mode is composed of an equal superposition of the two atomic excited states and a photonic component that vanishes as the magnetic field approaches zero.

By applying a magnetic field $B$, we can mix photonic character into the dark mode, inducing transmission (also referred to as transparency) for probe light near $\omega_{0}$. The probe light is horizontally polarized and is perpendicular to the vertically oriented magnetic field. Each trace in Fig. 2(a) corresponds to a different applied magnetic field with strength parametrized by the induced Zeeman frequency splitting $\Delta / 2 \pi=B(2.1 \mathrm{MHz} / \mathrm{G})$.

To describe the system, we extend the linearized inputoutput equations of Ref. [31] to include an additional atomic transition written in a rotating frame at the average atomic transition frequency $\omega_{0}$ as (a)

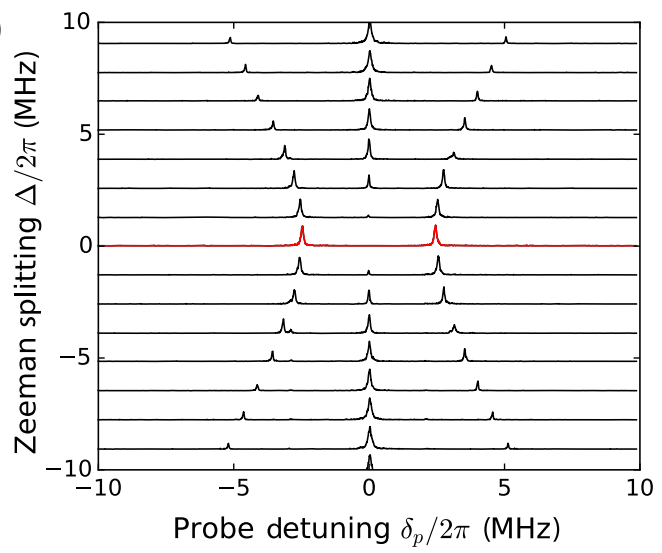

(b)

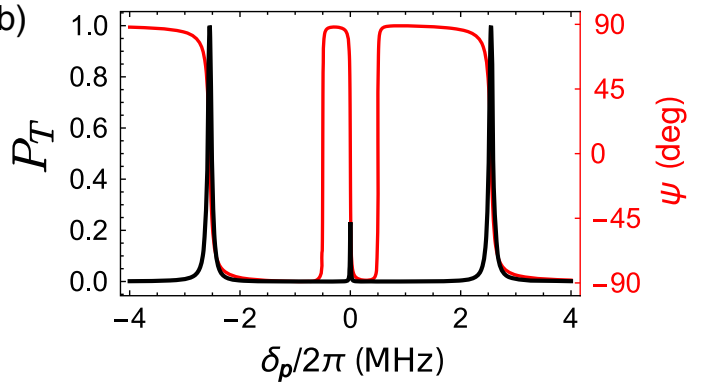

FIG. 2. (a) The transmitted power $P_{T}$ through the cavity versus the probe detuning $\delta_{p}$, with $\delta_{c}=0$. Each trace was taken for different applied magnetic fields, creating different Zeeman splittings $\Delta$ labeled on the vertical. The central red trace is taken for $\Delta=0$ and displays a collective vacuum Rabi splitting $\Omega / 2 \pi=5 \mathrm{MHz}$. When a magnetic field is applied perpendicular to the probe polarization, inducing a Zeeman splitting $\Delta$, a new transmission feature appears between the two original resonances of the vacuum Rabi splitting. (b) Linearized theory showing the power $P_{T}$ and phase $\psi$ of the transmitted light, plotted here for $\Omega / 2 \pi=5 \mathrm{MHz}$ and $\Delta / 2 \pi=1 \mathrm{MHz}$.

$\dot{a}=-\frac{1}{2}(\gamma+\imath \Delta) a-\imath \frac{1}{2 \sqrt{2}} \Omega c$,

$\dot{b}=-\frac{1}{2}(\gamma-\imath \Delta) b-\imath \frac{1}{2 \sqrt{2}} \Omega c$,

$\dot{c}=-\frac{1}{2}\left(\kappa+\imath 2 \delta_{c}\right) c-\imath \frac{1}{2 \sqrt{2}} \Omega(a+b)+\sqrt{\kappa_{1}} c_{i} e^{\imath \delta_{p} t}$.

Here, $\delta_{c}=\omega_{c}-\omega_{0}$ is the detuning of the cavity-resonance frequency $\omega_{c}$ from atomic resonance, $\Omega$ is the observed collective vacuum Rabi splitting when $\Delta=0, \gamma$ is the decay rate of the excited atomic states, $\kappa$ is the cavity power decay rate, and $\kappa_{1}$ is the coupling of the input cavity mirror that is driven by an externally incident probe field with complex amplitude $c_{i}$ at probe frequency $\omega_{p}$ detuned from atomic resonance by $\delta_{p}=\omega_{p}-\omega_{0}$. The complex variables $a=\langle\hat{a}\rangle, b=\langle\hat{b}\rangle$, and $c=\langle\hat{c}\rangle$ are expectation values of bosonic lowering operators describing the cavity $c$ and collective excitations of the two atomic transitions $a$ and $b$. The required Holstein-Primakoff approximation assumes 
weak excitation such that the number of atoms in the excited states $M_{a}=|a|^{2}, M_{b}=|b|^{2} \ll N$ is a small fraction of the total atom number $N$. The average number of photons in the cavity is given by $M_{c}=|c|^{2}$, and the complex field transmitted through the cavity is $c_{t}=\sqrt{\kappa_{2}} c$, with $\kappa_{2}$ being the coupling of the output mirror. The transmitted probe power relative to the incident probe power is $P_{T}=\left|c_{t} / c_{i}\right|^{2}$, and the relative phase is $\psi=\arg \left(c_{t} / c_{i}\right)$.

Figure 2(b) shows the calculated steady-state transmitted power and phase for a single Zeeman splitting. The phase response changes rapidly near zero probe detuning, which results in a narrow MIT resonance compared to the broad vacuum Rabi splitting or bright modes for which the phase changes more slowly.

In order to describe the linewidth of the dark state resonance, we introduce a mixing angle $\theta$ defined by $\sin ^{2} \theta=\bar{\Delta}^{2} /\left(\Omega^{2}+\bar{\Delta}^{2}\right)$. Here, the effective detuning is $\bar{\Delta}^{2}=\Delta^{2}+\gamma^{2}$. The character of the dark state excitation is given by the ratio of the probability that the excitation is photoniclike $P_{c}=M_{c} /\left(M_{c}+M_{a}+M_{b}\right)=\sin ^{2} \theta$ versus atomiclike $P_{a b}=\left(M_{a}+M_{b}\right) /\left(M_{c}+M_{a}+M_{b}\right)=\cos ^{2} \theta$. The dark state excitation can decay into free space at rate $R_{a b}$ or by emission through the cavity mirrors $R_{c}$, with the ratio of the rates given simply by $R_{a b} / R_{c}=\gamma /\left(\kappa \tan ^{2} \theta\right)=$ $N C(\gamma / \bar{\Delta})^{2}$, where the single particle cooperativity parameter is $C=4 g^{2} / \kappa \gamma$.

The linewidth of the dark state resonance can be written as

$$
\kappa^{\prime}=\left(\gamma \cos ^{2} \theta+\kappa \sin ^{2} \theta\right) / b .
$$

The term in parentheses is a weighted average of atom and cavity linewidths that reflects the character of the mode. The correction factor is $b=d \cos ^{2} \theta+\sin ^{2} \theta$, where $d=\left(\Delta^{2}-\gamma^{2}\right) / \bar{\Delta}^{2}$. When $\Delta \gg \gamma$, both $b$ and $d$ approach unity. At small detunings $\Delta \sim \gamma$, the responses of the dark and bright modes to the applied drive become comparable, causing a modification of the correction factor. In the regime experimentally explored here $(b \approx 1), \kappa^{\prime}$ is simply the full width at half maximum linewidth of the power transmission feature. To define a linewidth as valid in general, we define the linewidth via $\kappa^{\prime}=2$ $\left.\left(d \psi / d \delta_{p}\right)^{-1}\right|_{\delta_{c}=\delta_{p}=0}$. For $\Omega \gg \Delta \gg \gamma$, the mixing angle is small and the linewidth approaches the atomic linewidth $\kappa^{\prime} \approx \gamma$, which can be much narrower than the cavity linewidth $\kappa$.

We measure the linewidth for the central dark resonance by linearly sweeping the probe laser's frequency over the cavity resonance and recording a time trace of power transmitted on a single photon counting module. A Lorentzian is fit to the central feature to extract the full width at half maximum. This measurement is taken for a range of different Zeeman splittings and vacuum Rabi splittings by varying the applied static magnetic field and atom number, respectively. Figure 3(a) shows collected data plotted against the
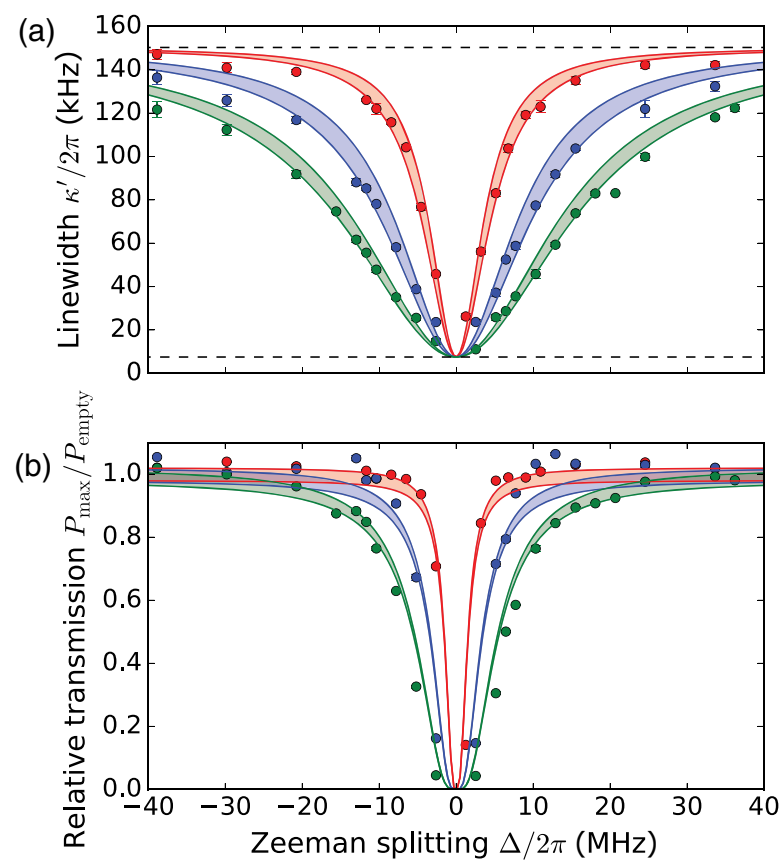

FIG. 3. (a) The measured linewidth of the central MIT transmission feature versus the induced Zeeman splitting between excited states. The traces are taken for three different collective vacuum Rabi frequencies $\Omega / 2 \pi=4.6(5)$ (red), 10(1) (blue), and 16(1) (green) $\mathrm{MHz}$, with values set by changing the total atom number $N$. The upper dashed line is the empty cavity's linewidth $\kappa$, and the lower dashed line is the atomic transition's linewidth $\gamma$. The minimum observed linewidth was $11 \mathrm{kHz}$. The shaded regions are no-free parameter predictions from the linearized model introduced in the text, indicating the \pm 1 standard deviation uncertainty bands based on independent measurements of $\Omega$. (b) The measured peak transmitted power of the central MIT transmission feature for the same collective Rabi frequencies. Here, the transmitted power is normalized to the peak transmitted power when the cavity is empty. Again, the shaded regions indicate the \pm 1 standard deviation uncertainty bands for the predictions.

theoretical prediction at several Rabi frequencies. For very small $\Delta$ 's the feature becomes increasingly narrow, approaching the atomic transition linewidth. For very large $\Delta$ 's, the feature linewidth approaches the cavity linewidth $\kappa$.

Figure 3(b) shows the peak transmitted power at the MIT feature's resonance for the same data shown in Fig. 3(a). The linearized theory predicts that the peak transmitted power is given by

$$
P_{\max }=\frac{4 \kappa_{1} \kappa_{2}}{\kappa^{2}} \frac{1}{\left(1+\frac{\gamma}{\kappa \tan ^{2} \theta}\right)^{2}} .
$$

Note that the term in the denominator above is the ratio of excitation decay rates $R_{a b} / R_{c}$. For large detunings $\Delta \gg \Omega$, $\gamma$, the peak transmission goes to that of an empty cavity $P_{\max } \rightarrow P_{\text {empty }}=4 \kappa_{1} \kappa_{2} / \kappa^{2}$.

In the regime experimentally explored here, a change in the cavity-resonance frequency $\omega_{c}$ by $\Delta \omega_{c}$ leads to a change in the dark state resonance frequency $\omega_{D}$ by a 


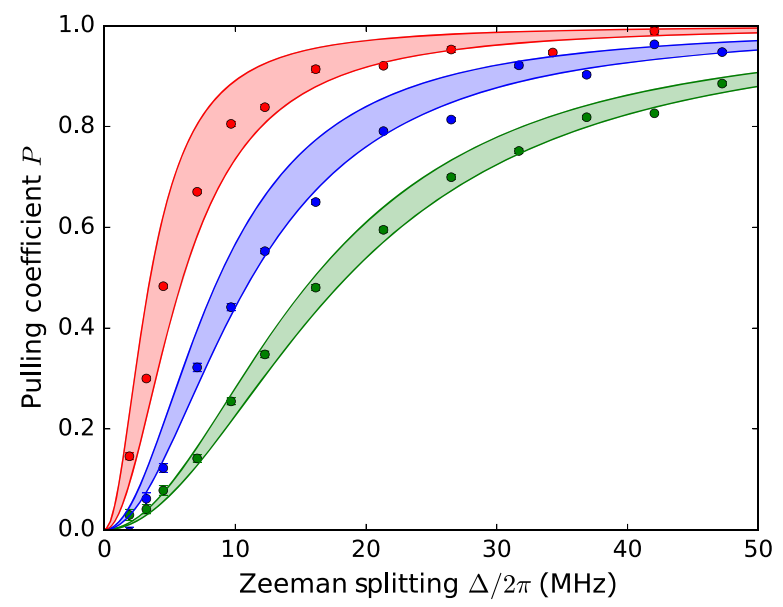

FIG. 4. The pulling coefficient $P$ versus Zeeman splitting $\Delta$ for several collective vacuum Rabi frequencies $\Omega / 2 \pi=5(1)$ (red), 10(1) (blue), and 17(1) (green) MHz. The prediction from the linearized theory is shown with \pm 1 standard deviation bands.

much smaller amount $\Delta \omega_{D}$. The pulling coefficient $P=$ $\Delta \omega_{D} / \Delta \omega_{c}$ expresses this ratio. A small pulling coefficient $(P \ll 1)$ is desirable for a frequency reference, as it will be less sensitive to thermal fluctuations and technical noise on the reference cavity. We can extract a pulling coefficient applicable to all parameter regimes from the linearized theory by considering how much the probe and cavity detunings would have to change to create equal changes in the quadrature amplitude of the transmitted field (such as one might measure using homodyne detection). This general pulling coefficient can be expressed as

$$
P=\frac{\sin ^{2} \theta}{b} .
$$

In the typical regime of operation $(b \approx 1)$, this is simply the photoniclike fraction of the dark excitation.

In Fig. 4, we show the measured pulling coefficient versus splitting $\Delta$ for several values of $\Omega$, along with the predicted pulling coefficients from the linearized theory. The pulling coefficients were measured by sweeping the probe laser frequency across the dark resonance and fitting the center frequency $\omega_{D}$ with a Lorentzian fit model. This is then repeated while toggling $\omega_{c}$ between two values separated by $100 \mathrm{kHz}$, and the pulling coefficient is determined from the change in $\omega_{D}$ versus $\omega_{c}$. Our lowest measured pulling coefficients are below $P=0.05$.

In principle, $\Delta$ can be reduced further to reach a smaller pulling coefficient, at the expense of transmitted power. The theoretical pulling coefficient reaches a minimum value of $P=1 /\left[1+N /\left(8 m_{c}\right)\right]$ at $\Delta=\sqrt{3} \gamma$, where $m_{c}=[\gamma /(2 g)]^{2}$ is the so-called critical photon number. The critical photon number is proportional to the cavity mode volume and atomic linewidth, but it does not depend on the mirror reflectivity. As a result, small pulling coefficients are reached by working with small cavity volumes and very narrow linewidth transitions. For spectroscopic applications, one would want to balance the desire for a low pulling coefficient against the need to collect transmitted photons without inducing heating in the atomic ensemble due to free-space scattering. The optimal parameter regime will depend on the specific requirements of the system.

While the majority of this work has been done with the atoms trapped in the Lamb-Dicke regime (i.e., confined to much less than the wavelength of the probe light) with respect to the cavity axis, we have also performed scans of the cavity transmission spectrum in which the atoms were unconfined along the cavity axis. In this configuration, the rms Doppler shift along the cavity axis is roughly $45 \mathrm{kHz}$. Despite this inhomogeneous broadening, we observe a center feature linewidth of $18.5 \mathrm{kHz}$, which we believe is limited by technical noise on the cavity frequency that arises when we turn down the lattice depth to release the atoms. We expect the linewidth of the dark feature to be insensitive to inhomogeneous broadening so long as $\Delta$ is much larger than the inhomogeneous broadening [32]. This insensitivity to Doppler broadening may make such techniques suitable to continuously operating atomic beam experiments, where confining the atoms to the Lamb-Dicke regime would be challenging.

To summarize, we have demonstrated a technique to realize a narrow spectroscopic feature based on a collective interaction between an ensemble of atoms and a highfinesse optical cavity. The center frequency of the feature can be made highly insensitive to changes in cavityresonance frequency. In analogy to EIT, this technique may also be applicable to tasks relevant for information processing. From time reversal symmetry arguments and the hierarchy $\Omega \gg \kappa \gg \gamma$, the theoretical maximum storage and retrieval efficiencies $P_{R}$ are given approximately by $P_{R} \approx 1-(\pi / 2)(\gamma / \Omega)$ [33]. This should be compared to a theoretical maximum efficiency $P_{R} \approx 1-\kappa \gamma / \Omega^{2}$ for the more typical assumptions $\kappa \gg \Omega, \Omega^{2} \gg \kappa \gamma$, and that the storage state does not decay [35].

We acknowledge contributions to the experimental apparatus by Karl Mayer, and also the helpful discussions with Anders S. Sørenson and Alexey V. Gorshkov. All of the authors acknowledge financial support from Defense Advanced Research Projects Agency (DARPA) QuantumAssisted Sensing and Readout (QuASAR), Army Research Office (ARO), National Science Foundation Physics Frontiers Centers (NSF PFC), and National Institute of Standards and Technology (NIST). J. R. K. C. acknowledges financial support from National Science Foundation Graduate Research Fellowship Program (NSF GRFP). This work is supported by the National Science Foundation under Grant No. 1125844.

[1] T. Kessler, C. Hagemann, C. Grebing, T. Legero, U. Sterr, F. Riehle, M. Martin, L. Chen, and J. Ye, A sub-40-mHzlinewidth laser based on a silicon single-crystal optical cavity, Nat. Photonics 6, 687 (2012). 
[2] B. Bloom, T. Nicholson, J. Williams, S. Campbell, M. Bishof, X. Zhang, W. Zhang, S. Bromley, and J. Ye, An optical lattice clock with accuracy and stability at the $10^{-18}$ level, Nature (London) 506, 71 (2014).

[3] N. Hinkley, J. Sherman, N. Phillips, M. Schioppo, N. Lemke, K. Beloy, M. Pizzocaro, C. Oates, and A. Ludlow, An atomic clock with $10^{-18}$ instability, Science 341, 1215 (2013).

[4] I. Ushijima, M. Takamoto, M. Das, T. Ohkubo, and H. Katori, Cryogenic optical lattice clocks, Nat. Photonics 9, 185 (2015).

[5] T. Rosenband, D. Hume, P. Schmidt, C. Chou, A. Brusch, L. Lorini, W. Oskay, R. Drullinger, T. Fortier, J. Stalnaker et al., Frequency ratio of $\mathrm{Al}^{+}$and $\mathrm{Hg}^{+}$single-ion optical clocks; metrology at the 17 th decimal place, Science 319, 1808 (2008).

[6] S. Kolkowitz, I. Pikovski, N. Langellier, M. D. Lukin, R. L. Walsworth, and J. Ye, Gravitational wave detection with optical lattice atomic clocks, Phys. Rev. D 94, 124043 (2016).

[7] P. W. Graham, J. M. Hogan, M. A. Kasevich, and S. Rajendran, New Method for Gravitational Wave Detection with Atomic Sensors, Phys. Rev. Lett. 110, 171102 (2013).

[8] A. Derevianko and M. Pospelov, Hunting for topological dark matter with atomic clocks, Nat. Phys. 10, 933 (2014).

[9] S. Blatt, A. Ludlow, G. Campbell, J. W. Thomsen, T. Zelevinsky, M. Boyd, J. Ye, X. Baillard, M. Fouché, R. Le Targat et al., New Limits on Coupling of Fundamental Constants to Gravity Using ${ }^{87} \mathrm{Sr}$ Optical Lattice Clocks, Phys. Rev. Lett. 100, 140801 (2008).

[10] C.-W. Chou, D. Hume, T. Rosenband, and D. Wineland, Optical clocks and relativity, Science 329, 1630 (2010).

[11] K. Numata, A. Kemery, and J. Camp, Thermal-Noise Limit in the Frequency Stabilization of Lasers with Rigid Cavities, Phys. Rev. Lett. 93, 250602 (2004).

[12] M. J. Martin, D. Meiser, J. W. Thomsen, J. Ye, and M. J. Holland, Extreme nonlinear response of ultranarrow optical transitions in cavity QED for laser stabilization, Phys. Rev. A 84, 063813 (2011).

[13] P. G. Westergaard, B. T. R. Christensen, D. Tieri, R. Matin, J. Cooper, M. Holland, J. Ye, and J. W. Thomsen, Observation of Motion-Dependent Nonlinear Dispersion with Narrow-Linewidth Atoms in an Optical Cavity, Phys. Rev. Lett. 114, 093002 (2015).

[14] B. T. R. Christensen, M. R. Henriksen, S. A. Schäffer, P. G. Westergaard, D. Tieri, J. Ye, M. J. Holland, and J. W. Thomsen, Nonlinear spectroscopy of Sr atoms in an optical cavity for laser stabilization, Phys. Rev. A 92, 053820 (2015).

[15] J. G. Bohnet, Z. Chen, J. M. Weiner, D. Meiser, M. J. Holland, and J. K. Thompson, A steady-state superradiant laser with less than one intracavity photon, Nature (London) 484, 78 (2012).

[16] M. A. Norcia and J. K. Thompson, Cold-Strontium Laser in the Superradiant Crossover Regime, Phys. Rev. X 6, 011025 (2016).

[17] M. A. Norcia, M. N. Winchester, J. R. K. Cline, and J. K. Thompson, Superradiance on the millihertz linewidth strontium clock transition, Sci. Adv. 2, e1601231 (2016).
[18] D. Meiser, J. Ye, D. R. Carlson, and M. J. Holland, Prospects for a Millihertz-Linewidth Laser, Phys. Rev. Lett. 102, 163601 (2009).

[19] K.-J. Boller, A. Imamoğlu, and S. E. Harris, Observation of Electromagnetically Induced Transparency, Phys. Rev. Lett. 66, 2593 (1991).

[20] M. Mücke, E. Figueroa, J. Bochmann, C. Hahn, K. Murr, S. Ritter, C. J. Villas-Boas, and G. Rempe, Electromagnetically induced transparency with single atoms in a cavity, Nature (London) 465, 755 (2010).

[21] G. Hernandez, J. Zhang, and Y. Zhu, Vacuum Rabi splitting and intracavity dark state in a cavity-atom system, Phys. Rev. A 76, 053814 (2007).

[22] See Supplemental Material at http://link.aps.org/ supplemental/10.1103/PhysRevLett.118.263601, which includes Ref. [23], for a further discussion of the mapping between MIT and EIT.

[23] W. Hanle, Über magnetische Beeinflussung der Polarisation der Resonanzfluoreszenz, Z. Phys. 30, 93 (1924).

[24] L. V. Hau, S. E. Harris, Z. Dutton, and C. H. Behroozi, Light speed reduction to 17 metres per second in an ultracold atomic gas, Nature (London) 397, 594 (1999).

[25] C. Liu, Z. Dutton, C. H. Behroozi, and L. V. Hau, Observation of coherent optical information storage in an atomic medium using halted light pulses, Nature (London) 409, 490 (2001).

[26] R. Zhao, Y. Dudin, S. Jenkins, C. Campbell, D. Matsukevich, T. Kennedy, and A. Kuzmich, Long-lived quantum memory, Nat. Phys. 5, 100 (2009).

[27] T. Peyronel, O. Firstenberg, Q.-Y. Liang, S. Hofferberth, A. V. Gorshkov, T. Pohl, M. D. Lukin, and V. Vuletić, Quantum nonlinear optics with single photons enabled by strongly interacting atoms, Nature (London) 488, 57 (2012).

[28] Y. Dudin and A. Kuzmich, Strongly Interacting Rydberg Excitations of a Cold Atomic Gas, Science 336, 887 (2012).

[29] J. Ningyuan, A. Georgakopoulos, A. Ryou, N. Schine, A. Sommer, and J. Simon, Observation and characterization of cavity Rydberg polaritons, Phys. Rev. A 93, 041802 (2016).

[30] M. A. Norcia and J. K. Thompson, Strong coupling on a forbidden transition in strontium and nondestructive atom counting, Phys. Rev. A 93, 023804 (2016).

[31] Z. Chen, J. G. Bohnet, J. M. Weiner, K. C. Cox, and J. K. Thompson, Cavity-aided nondemolition measurements for atom counting and spin squeezing, Phys. Rev. A 89, 043837 (2014).

[32] R. Houdré, R. P. Stanley, and M. Ilegems, Vacuum-field Rabi splitting in the presence of inhomogeneous broadening: Resolution of a homogeneous linewidth in an inhomogeneously broadened system, Phys. Rev. A 53, 2711 (1996).

[33] See Supplemental Material at http://link.aps.org/ supplemental/10.1103/PhysRevLett.118.263601, which includes Ref. [34], for a further discussion of photon storage and retrieval efficiency.

[34] H. J. Kimble, Strong interactions of single atoms and photons in cavity QED, Phys. Scr. T76, 127 (1998).

[35] A. V. Gorshkov, A. André, M. D. Lukin, and A. S. Sørensen, Photon storage in $\Lambda$-type optically dense atomic media. I. Cavity model, Phys. Rev. A 76, 033804 (2007). 